\title{
La lumière pour une imagerie chimique des peintures
}

Laurence de Viguerie, Matthias Alfeld et Philippe Walter (philippe.walter@upmc.fr)

Sorbonne Universités, UPMC Université Paris 6, CNRS, UMR 8220

Laboratoire d'archéologie moléculaire et structurale (LAMS), 4 place Jussieu, 75005 Paris

L'utilisation des techniques d'imagerie chimique pour

l'étude des peintures d'art

est en plein essor.

Ces techniques non invasives

et mobiles permettent

I'acquisition d'un grand

nombre de données spatiales

et spectrales, dont

I'interprétation ouvre

de nombreuses perspectives

pour regarder autrement

les œuvres d'art.

Nous présentons ici

les concepts et les applications

de deux techniques :

I'imagerie par spectrométrie

de fluorescence $X$ et l'imagerie

hyperspectrale en lumière

visible et proche infrarouge.

Remerciements

Merci à Jacques et Marie-Hélène Fichefeux qui nous ont permis d'analyser Le marchand de fruits et à la Région Ile-de-France (DIM Analytics), pour son soutien au programme IMAPAT de construction par le LAMS d'un laboratoire mobile d'analyse non invasive pour le patrimoine.

\section{Évolution des techniques d'imagerie}

Les méthodes d'analyse des œuvres d'art ont considérablement évolué depuis les premiers laboratoires dédiés aux matériaux du patrimoine, bénéficiant des avancées techniques de nombreux domaines et d'un intérêt croissant de la communauté scientifique mais aussi du grand public. Déjà en 1959, Madeleine Hours (alors responsable du Laboratoire de recherche des musées de France, situé au Louvre) fascine les téléspectateurs en leur révélant « la double vie des chefsd'œuvre » par l'examen de ces tableaux de grands maitres sous divers rayonnements : rayons $\mathrm{X}$ et lumière rasante sont utilisés pour découvrir et raconter leur histoire secrète.

Aujourd'hui, la constitution d'un dossier d'imagerie scientifique est une étape de routine avant une restauration ou lors d'une analyse de l'œuvre. Ces images photographiques et radiographiques renseignent sur l'œuvre et son histoire, de sa création à nos jours : la radiographie a été la première technique non destructive permettant d'obtenir une information sur les couches sous-jacentes d'une peinture. D'autres techniques, basées sur des rayonnements moins énergétiques, donnent des informations à différentes profondeurs. Ainsi, la réflectographie infrarouge et la photographie sous rayonnement ultraviolet (en réalité photographie de fluorescence induite par les UV) sont utilisées comme moyens d'investigation pour la peinture de chevalet (voir l'article de L. Beck dans ce numéro, p. 100).

Les propriétés particulières de certains pigments vis-à-vis de ces rayonnements (ultraviolet ou infrarouge) permettent de mettre en évidence leur utilisation. Ainsi, une photographie en lumière infrarouge distingue des pigments de natures chimiques différentes ayant une couleur très similaire en lumière visible. Pour une lecture plus facile de la photographie sous infrarouge (en échelle de gris), une image en fausses couleurs combinant l'image de l'œuvre dans le visible et la photographie sous infrarouge est souvent utilisée. De la même façon, la photographie en ultraviolet par réflexion, qui consiste à enregistrer l'ultraviolet réfléchi par la couche picturale soumise à un rayonnement UV, est souvent exploitée sous forme d'images en fausses couleurs. L'utilisation récente de ce type d'image permet non seulement l'analyse visuelle de l'œuvre et de son histoire, mais aussi la détermination de la nature de certains pigments à partir de bases de données de référence.

Parallèlement au développement des techniques d'imagerie, l'analyse chimique ponctuelle a connu de grandes avancées, et des techniques comme la fluorescence des rayons $\mathrm{X}$ ou la spectroscopie infrarouge sont désormais utilisées en routine pour l'analyse des matériaux du patrimoine. Le défi actuel est de combiner ces deux aspects pour obtenir une imagerie chimique des œuvres.

\section{Imagerie par spectrométrie de fluorescence des rayons $X$}

La spectrométrie de fluorescence des rayons $\mathrm{X}$ (XRF) est une technique d'analyse élémentaire quantitative basée sur l'émission de rayons $\mathrm{X}$, dits secondaires, consécutive à une excitation des couches atomiques profondes par un faisceau de rayons $\mathrm{X}$, dit primaire. Le 


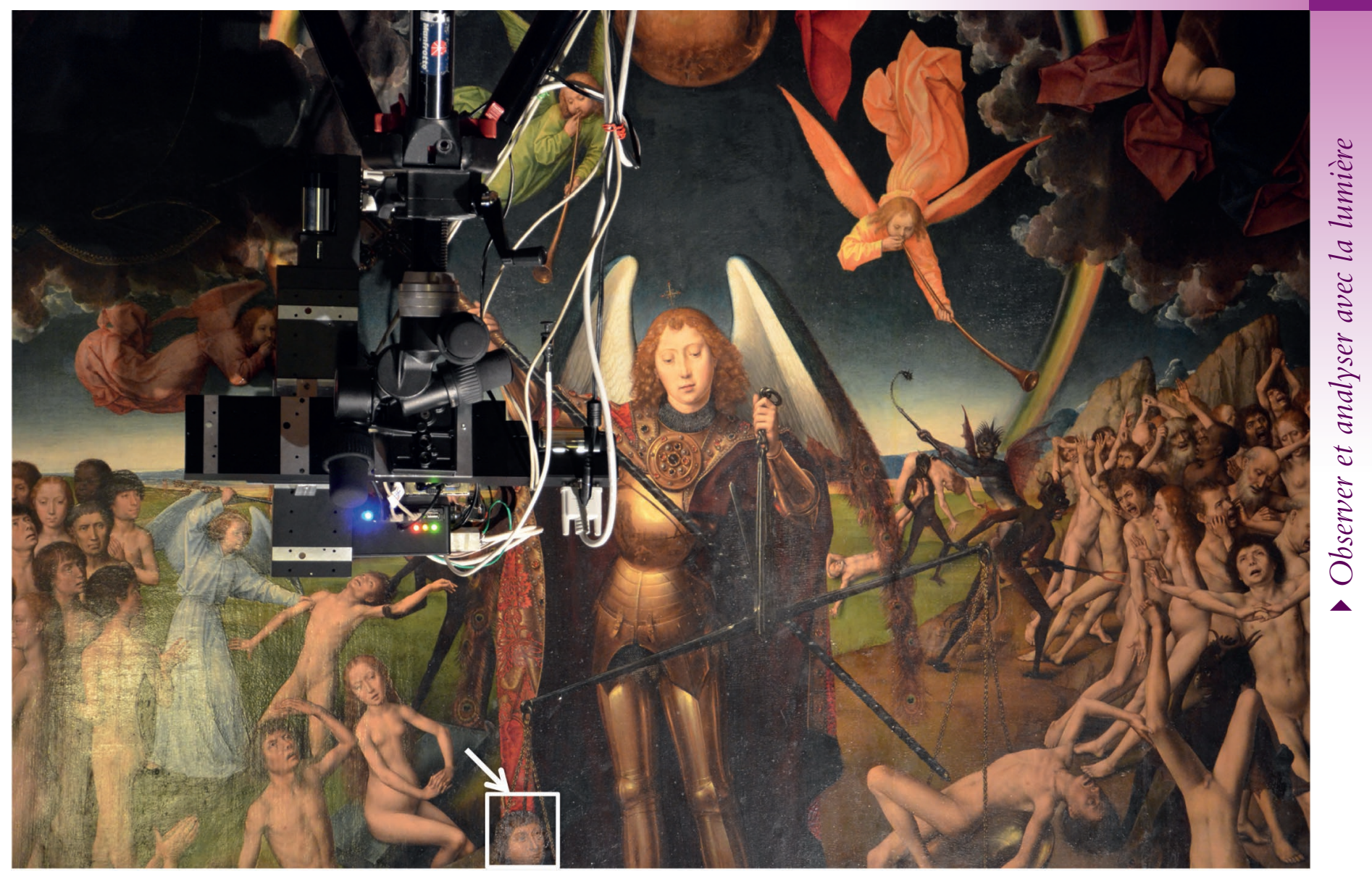

1. L’imageur par spectrométrie de fluorescence X lors de l'étude du retable du Jugement dernier de Hans Memling, au Musée National à Gdansk.

rayonnement émis est caractéristique de la nature et de l'abondance des éléments présents. Il s'agit d'une méthode simple, rapide et non destructive, qui est à ce titre très couramment utilisée pour l'analyse des matériaux du patrimoine.

L'imagerie par fluorescence des rayons X s'est développée rapidement et permet de mesurer la distribution des pigments présents à la surface mais aussi dans les couches sous-jacentes, suivant la profondeur de pénétration des rayons $\mathrm{X}$. Il s'agit d'imager la surface de l'échantillon en le balayant par un faisceau de rayons $\mathrm{X}$ de dimensions millimétriques, et d'analyser ensuite le rayonnement de fluorescence émis.

Cette méthode s'est révélée très utile pour la recherche sur les peintures artistiques : les éléments chimiques détectés peuvent parfois révéler des couches sous-jacentes cachées, montrant les modifications que l'artiste ou des restaurations postérieures ont apporté. Elle ouvre des possibilités pour les historiens d'art d'explorer plus en détail les œuvres, leur histoire et leur processus de création [1].

Ce type d'analyse est décrit pour la première fois au début des années 1990 , mais cette méthode a vraiment connu son essor en révélant un portrait de paysanne caché sous une seconde peinture de Van Gogh représentant un champ de blé [2]. Dans ces premières expériences réalisées sous rayonnement synchrotron, la peinture étudiée est montée sur un support motorisé et déplacée devant le faisceau. Ce type de montage, qui limite la taille des peintures étudiées, est coûteux et nécessite de nombreux efforts logistiques : il n'est pas aisé de déplacer une œuvre de grand maître dans l'enceinte d'un synchrotron ! Aujourd'hui, des instruments permettant une analyse in situ des œuvres sont privilégiés. Pour cela, tubes et détecteurs sont montés sur des translations motorisées et se déplacent devant la peinture, qui, elle, reste immobile (fig. 1).

L'interprétation des images et spectres obtenus ne peut se faire sans prendre en compte la nature complexe des œuvres analysées : une peinture est réalisée en superposant un certain nombre de couches, qui peuvent être inhomogènes en concentration et en épaisseur. Cette superposition permet de créer certains effets de couleur, de transparence ou de profondeur. Lors de l'analyse, les rayons $\mathrm{X}$ les plus énergétiques pénètrent en profondeur la matière, tandis que ceux de plus faible énergie n'interagissent qu'avec les couches superficielles. De la même façon, le rayonnement provenant des couches profondes est absorbé par les couches superficielles suivant son énergie : un vernis de $40 \mu \mathrm{m}$ sur une couche d'orpiment $\mathrm{As}_{2} \mathrm{~S}_{3}$ absorbera environ $38 \%$ de l'intensité du signal du soufre (à 2,3 keV), mais seulement $1 \%$ de celle du signal de l'arsenic (à 10,54 keV). Un raisonnement basé sur les phénomènes d'absorption des couches superficielles permet d'obtenir des informations sur la stratigraphie de la peinture; il est en tout cas nécessaire de les considérer pour ne pas parvenir à des conclusions erronées. Le traitement des données par le logiciel libre PyMca (http://pymca.sourceforge.net) prend en compte ces contributions par une simulation " multicouche " du spectre [3]. Chaque cas d'étude est cependant différent; il n'est pas facile de généraliser cette approche qui ne concerne, tout au plus, que les 50 à 100 premiers micromètres du tableau.

L'étude de la peinture de Hans Memling, Le Jugement dernier, achevée en 1471 (triptyque exposé au Musée National à Gdansk, fig. 1), révèle un aspect technique 
tout à fait exceptionnel de l'art de cette époque. Le portrait du personnage placé sous les ailes de l'archange Michel (cadre blanc), a été peint sur une feuille d'étain qui a ensuite été collée, comme un insert, sur la surface du tableau. Ce portrait représente Tommaso Portinari, commanditaire final de l'œuvre, qui avait remplacé Angelo Tani à la tête de la banque des Médicis à Bruges en 1471.

La cartographie par spectrométrie de fluorescence des rayons $\mathrm{X}$ de cette zone (fig. 2) révèle, par le signal des raies $\mathrm{K}$ de l'étain (SnK), la forme de la feuille, ajustée à la dimension de la tête. Concernant les raies L de l'étain $(\mathrm{SnL})$, de plus faible énergie, l'absorption des rayons $\mathrm{X}$ à travers les couches de peinture du visage et des cheveux, riches en blanc de plomb, modifie fortement leur distribution. On remarque également que différentes teintes ont été obtenues en ajoutant plus ou moins de vermillon $\mathrm{HgS}$ (cartographie de $\mathrm{Hg}$ ), ainsi que des terres naturelles riches en fer.

Cette pratique de l'incrustation sur la peinture d'un visage préparé sur un support d'étain est rare. Elle a également été mise en œuvre par Rogier van der Weyden pour Le Triptyque des sept sacrements, conservé au Musée Royal des Beaux-Arts d'Anvers. L'artiste pouvait ainsi sans doute se rendre chez son commanditaire pour peindre son portrait, sans lui demander de venir dans son atelier.

\section{Imagerie spectrale visible et proche infrarouge}

L'imagerie hyperspectrale consiste à acquérir des informations spatiales et spectrales sur un objet : à chaque pixel de l'image, un spectre de réflectance de la lumière (pourcentage $\mathrm{R}(\boldsymbol{\lambda})$ de lumière réfléchie en fonction de la longueur d'onde $\lambda$ de la lumière incidente) est mesuré de manière continue, avec une grande résolution spectrale dans une gamme de longueurs d'onde donnée correspondant aux domaines du visible et du proche infrarouge.

Utilisée tout d'abord pour la télédétection, pour la géologie ou l'astronomie, l'imagerie spectrale a ensuite trouvé des applications dans de nombreux domaines [4]. Pour la peinture et les manuscrits, elle a été développée en premier lieu afin d'augmenter la fidélité de la couleur des images obtenues. Au départ, son exploitation spectrale se limitait à des comparaisons qualitatives d'une longueur d'onde à une autre, et permettait essentiellement d'identifier des zones de compositions différentes, la dégradation de matériaux, la présence de dessins préparatoires. Aujourd'hui, avec un nombre croissant de bandes spectrales disponibles et une rapidité importante de la mesure, les spectres de réflectance sont utilisés en tant que tels, pour l'identification de pigments ou même de liants. Ce mode d'imagerie peut être employé en UV, visible, proche infrarouge ou fluorescence UV. Les images sont utilisées et manipulées afin d'obtenir des réflectogrammes dans le domaine proche infrarouge, de meilleur contraste, plus lisible ; mais il s'agit surtout d'un véritable outil d'analyse, qui permet l'acquisition de milliers de spectres en un scan.

L'interprétation des spectres de réflectance $\operatorname{R}(\lambda)$ dans la gamme spectrale du visible permet l'identification d'un grand nombre de pigments. En effet, le spectre de réflectance n'est pas juste une indication de la couleur observée mais bien une signature du phénomène à l'origine de la couleur. L'absorption de la lumière visible est due à différents mécanismes de transitions électroniques (transferts de charge, transitions électroniques entre orbitales...). À titre d'exemple, sont présentés dans l'encadré 1 les spectres de réflectance obtenus après acquisition de l'image hyperspectrale d'une palette de gouache (fig. 3).
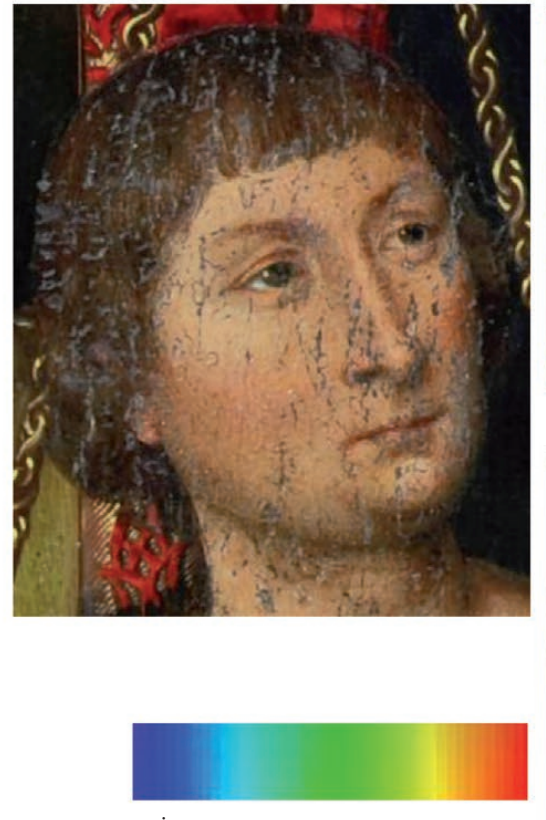

$\min$

$\max$

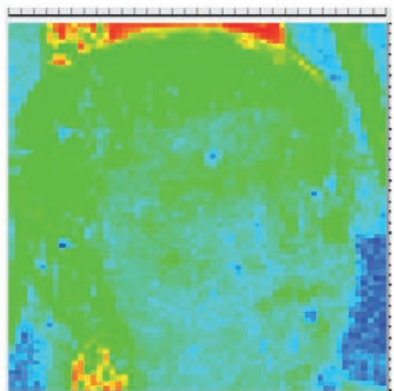

$\mathrm{PbL}$

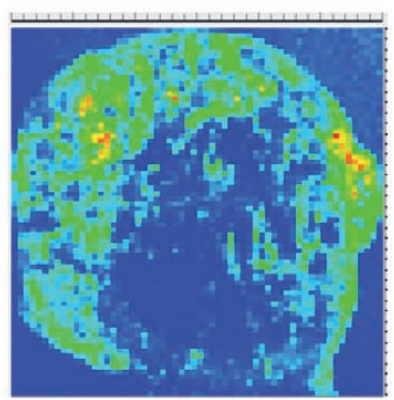

SnL

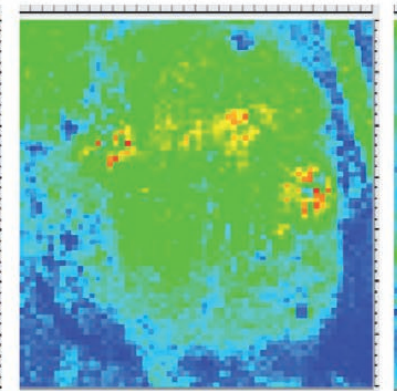

$\mathrm{PbM}$

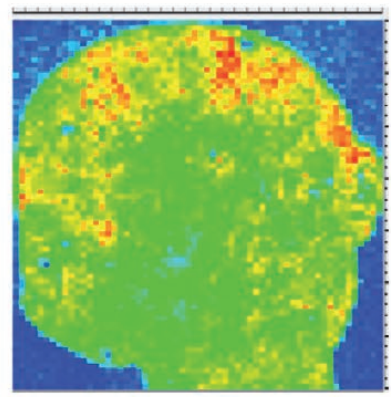

SnK

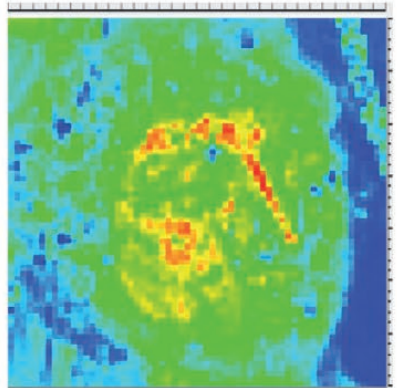

$\log (\mathrm{HgL})$

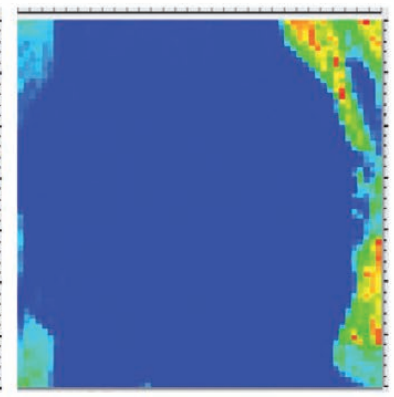

CuK

2. Cartographie par spectrométrie de fluorescence des rayons X du visage de Tommaso Portinari, peint sur une feuille d'étain par Hans Memling. Images en fausses couleurs des raies d'émission de Pb, Hg, Sn et Cu. Image $60 \times 70$ pixels (pas de $1 \mathrm{~mm}$, et $1 \mathrm{~s}$ d'acquisition par point mesuré). 

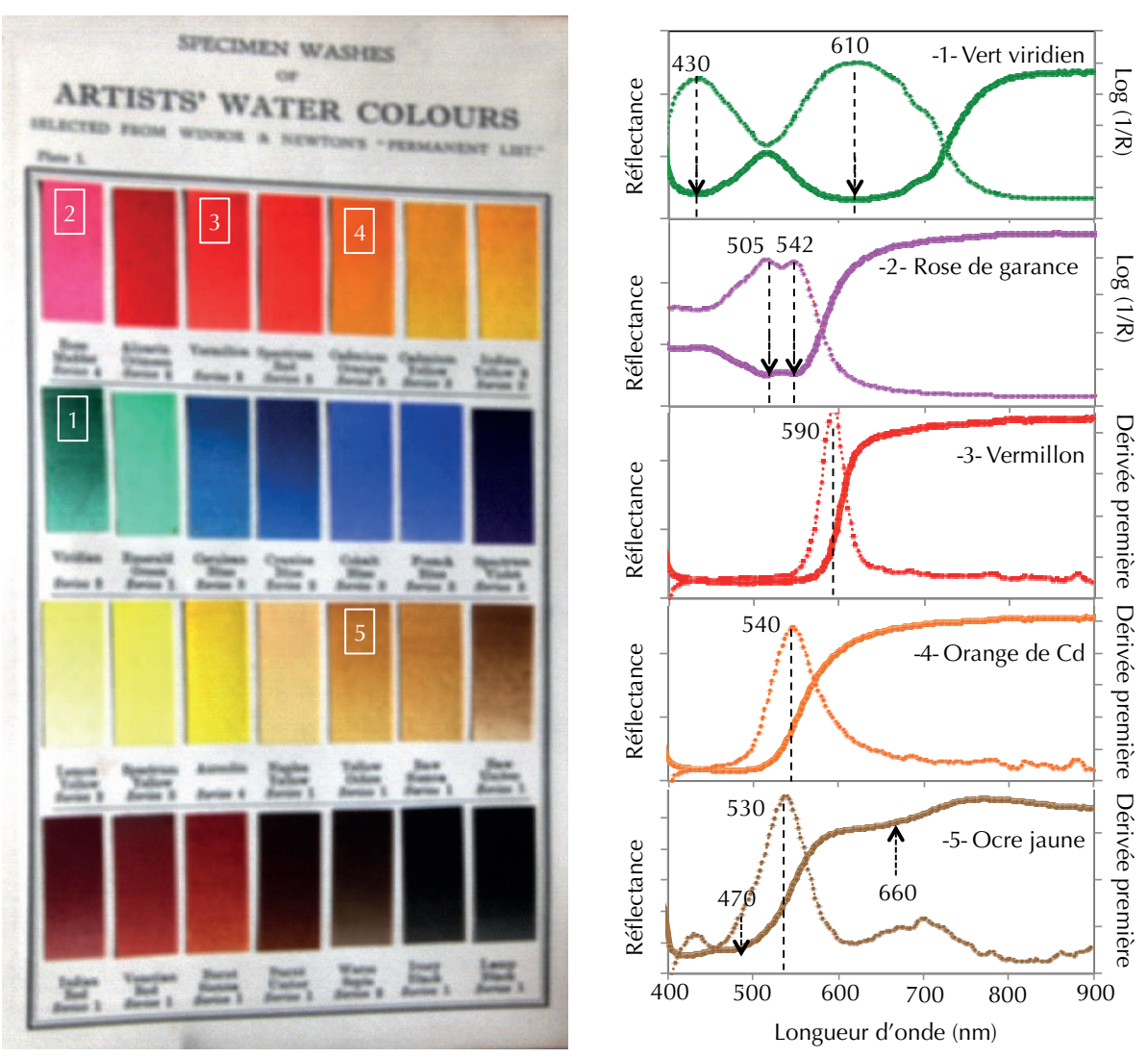

3. Spectres de réflectance de cinq pigments d'une palette de gouache, dans le domaine de longueur d'onde 400-900 nm.

À gauche : image de la palette, avec 1600 × 1402 spectres mesurés (acquisition des données en 140 secondes par spectre). L'image correspond à la superposition de trois images, acquises à trois longueurs d'onde différentes et affectées aux trois couleurs primaires : ici, les trois coordonnées $\mathrm{R}, \mathrm{G}$ et $\mathrm{B}$ correspondent respectivement aux réflectances à 628, 542 et $462 \mathrm{~nm}$. Les encadrés blancs représentent les régions d'intérêt analysées.

À droite : spectres obtenus (lignes épaisses continues) et leurs dérivées premières (lignes fines en pointillés).

(1) Le vert viridien $\mathrm{Cr}_{2} \mathrm{O}_{3} \cdot 2 \mathrm{H}_{2} \mathrm{O}$ est un pigment synthétique, mis au point par Vauquelin dans les années 1800. La couleur du vert viridien est caractéristique de l'ion $\mathrm{Cr}^{3+}$ en environnement octaédrique : deux transitions d-d apparaissent lors de l'éclatement du champ cristallin (qui correspond à une séparation des niveaux d'énergie des orbitales d), respectivement dans les régions $580-650 \mathrm{~nm}$ et $420-460 \mathrm{~nm}$.

(2) Le rose de garance est un colorant naturel composé de cycles aromatiques : des transitions électroniques dans les orbitales moléculaires délocalisées entraînent une absorption dans I'UV ou le visible. Le spectre de réflectance des colorants d'origine végétale, tel que la garance, montre une bande d'absorption avec deux maxima situés idéalement à 510-515 et 540-545 nm.

(3) Dans le cas du vermillon, le cinabre HgS est un semiconducteur dont le gap se situe à $2 \mathrm{eV}$; donc toutes les longueurs d'onde inférieures à $620 \mathrm{~nm}$ sont absorbées, d'où la couleur rouge du vermillon. La dérivée première du spectre de réflectance du vermillon présente un maximum entre 590 et 605 nm, ce qui permet de le différencier d'autres pigments semi-conducteurs.

(4) Le phénomène à l'origine de la couleur est le même pour l'orange de cadmium (avec un maximum de la dérivée à 540 nm).
(5) Les pigments à base d'oxyde de fer, tels que les ocres, doivent leur couleur à un mécanisme de transfert de charge du ligand (ions $\mathrm{O}^{2-}$ ou $\mathrm{OH}^{-}$) vers le cation $\mathrm{Fe}^{3+}$. C'est la différence d'environnement de l'ion ferrique qui est responsable de la différence de couleur entre la goethite $\alpha-\mathrm{FeOOH}$ (ocre jaune) et I'hématite $\alpha-\mathrm{Fe}_{2} \mathrm{O}_{3}$ (ocre rouge) [5]. Leurs spectres de réflectance sont caractérisés par une forme en $\mathrm{S}$ avec, dans le cas de la goethite, un front d'absorption entre 500 et $580 \mathrm{~nm}$, et une large bande d'absorption centrée autour de $660 \mathrm{~nm}$ (voir la figure).

Instrumentation. Le système d'imagerie hyperspectrale est composé d'une source lumineuse (lampe halogène) et d'une caméra hyperspectrale comprenant un spectrographe permettant la décomposition de la lumière en ses différentes composantes. Le spectrographe balaye l'objet ligne par ligne ; pour chaque ligne, une image matricielle est obtenue, composée d'un axe spatial et d'un axe spectral. Le système est translaté devant l'œuvre pour obtenir une image qui contient, en chaque pixel, un spectre avec une résolution spectrale de 2,8 $\mathrm{nm}$.

Dans notre cas, l'imageur VNIR de chez Specim [6] est composé d'un spectrographe V10E monté avec une caméra CCD (1600 × 1200 pixels). 


\section{\〉}

L'interprétation des spectres de réflectance est aisée dans le cas de cette palette simple, lorsque les pigments sont utilisés seuls et en une seule couche...

Dans le cas général, différents pigments sont utilisés et dispersés dans un liant, formant ainsi une couche picturale qui peut être recouverte d'un vernis et repose sur d'autres couches ou sur un support. De nombreux phénomènes interviennent alors (diffusion, réflexion et absorption), et une modélisation devient nécessaire pour en tenir compte et parvenir à interpréter le signal obtenu. La théorie de Kubelka-Munk est la plus utilisée ; dans le cas de couches de peintures opaques, la réflectance peut être exprimée sous une forme analytique simple en fonction du rapport des coefficients $K$, l'absorbance, et $S$, la diffusion à chaque longueur d'onde. Une simulation peut ainsi être obtenue pour des pigments de différentes concentrations, tailles de particules et dans différents liants.

Il s'agit cependant de traitements complexes, à réaliser sur un grand nombre de données ; ceci explique que l'interprétation des données d'imagerie hyperspectrale utilise rarement ce type de traitement quantitatif dans le cas des œuvres d'art. Les principaux résultats de la littérature s'appuient sur des comparaisons à des bases de données de référence très complètes et révèlent la potentialité de ces techniques, en particulier lorsque la gamme spectrale est étendue. Des groupes, notamment ceux de la National Gallery de Washington, pionniers dans le domaine, ont montré les possibilités ouvertes par l'utilisation combinée de deux caméras spectrales dans le visible et le proche IR. L'identification des pigments par cette technique a été démontrée par des mesures collectées jusqu'à $1700 \mathrm{~nm}$ sur une œuvre de Picasso (Arlequin musicien) [7]. Les spectres correspondant à ces matières ont été comparés avec des bases de données spectrales de référence et combinées non linéairement.

D'autres travaux ont montré ces dernières années les potentialités de l'analyse spectrale en proche infrarouge pour l'identification des liants, d'abord par des analyses ponctuelles sur une série de matériaux de référence puis par imagerie hyperspectrale, notamment sur des panneaux peints par Cosimo Tura (autour de 1475) avec une technique mixte à l'œuf et à la colle [8]. Il est nécessaire, dans ce cas, de considérer un domaine spectral d'analyse entre $800 \mathrm{~nm}$ et $2500 \mathrm{~nm}$, afin d'inclure dans l'étude certaines vibrations des groupes fonctionnels de carboxyles ou d'amides associés avec la matière organique. C'est là une avancée majeure dans le domaine, la question du liant ne pouvant jusqu'à présent être résolue que par prélèvement et analyse séparative.

\section{L'apport des analyses combinées}

La gamme d'utilisation et les applications possibles de chaque technique étant forcément limitées, il est souvent utile, voire nécessaire, de coupler plusieurs techniques d'analyse : cela permet d'élargir la palette des matériaux détectés et de mieux comprendre ou de confirmer les résultats obtenus.

Un exemple d'imagerie couplant rayons $\mathrm{X}$, visible et infrarouge, est donné dans l'encadré 2 sur un tableau attribué à l'entourage du peintre néerlandais Goltzius, de la fin du XVI ${ }^{\mathrm{e}}$ siècle, représentant un marchand de fruits (à droite sur le tableau) avec son client (fig. 4). Par fluorescence des rayons X (XRF), de nombreux pigments peuvent être identifiés par la présence d'éléments chimiques caractéristiques. Une couche sous-jacente de blanc de plomb est présente en tout point analysé (épaisseur estimée d'environ 40 microns). La lisibilité du tableau est aujourd'hui très altérée par la couche de vernis irrégulière et jaunie ; cependant, le tableau devait paraître bien plus coloré lors de sa réalisation.

- Des terres colorées, jaunes, rouges ou brunes, sont visibles, indiquées par la présence de fer dans de nombreuses zones du tableau (en bleu dans l'image en fausses couleurs XRF, seconde à partir de la gauche en haut de la figure 4). Dans les zones les plus sombres (fond du tableau), une terre d'ombre contenant une plus forte proportion de manganèse que de fer, a été utilisée.

- Les carnations sont obtenues par un mélange de blanc de plomb et de vermillon en petite quantité ; des terres ont aussi été ajoutées. La couleur rouge du ruban du chapeau du marchand correspond à du vermillon (sulfure de mercure $\mathrm{HgS}$ ).
- Un jaune de plomb et d'étain a été utilisé, mélangé à des terres, notamment pour la veste du personnage de gauche : l'étain (Sn) est visible en vert dans l'image en fausses couleurs XRF de la figure 4.

- Le vert de la pastèque et du manteau du marchand (observés sur la photographie de l'oeuvre) est obtenu par un pigment à base de cuivre $(\mathrm{Cu}$ est en rouge dans l'image en fausses couleurs XRF de la figure 4). Du cuivre est aussi présent en forte quantité dans les zones très sombres, presque noires, qui correspondent aux plumes et aux feuilles des deux chapeaux.

Par combinaison avec l'analyse hyperspectrale en proche infrarouge, nous avons pu préciser la nature de ce pigment au cuivre. Il ne s'agit pas de malachite comme la couleur verte le laissait présager, mais d'azurite. L'azurite $2 \mathrm{CuCO}_{3} \cdot \mathrm{Cu}(\mathrm{OH})_{2}$ et la malachite $\mathrm{CuCO}_{3} \cdot \mathrm{Cu}(\mathrm{OH})_{2}$, de composition proche mais de structure différente, sont en effet nettement différentiables en proche infrarouge. De manière générale, l'absorption des pigments inorganiques est importante dans le proche infrarouge lorsqu'ils présentent des combinaisons de bandes $\mathrm{OH}$ ou des transitions électroniques de faible énergie (composés de cobalt ou de fer). Le vermillon, par exemple, est complètement transparent dans cette gamme spectrale mais il est bien identifié, comme nous l'avons vu, dans le domaine visible. La spécificité limitée de la spectroscopie en proche infrarouge, moins sensible aux changements structuraux que l'infrarouge moyen, est un avantage pour l'identification des liants car elle permet d'utiliser en première approche des composés non vieillis comme matériaux de référence. Ici, l'ensemble des bandes observées sur le spectre de réflectance du vêtement du marchand (région (a) sur la figure 4) peut être attribué à une huile. Cette série de bandes se retrouve dans l'ensemble des zones analysées, ce qui indique l'utilisation d'une huile siccative (composée à l'origine principalement de triglycérides insaturés) comme liant, résultat attendu dans le cas d'une peinture sur bois de la fin du XVI ${ }^{\mathrm{e}}$ siècle. Ainsi, la combinaison de ces deux techniques d'imagerie par fluorescence $\mathrm{X}$ et en proche infrarouge a permis l'identification de la palette de pigments utilisée par le peintre, mais aussi de son liant. 

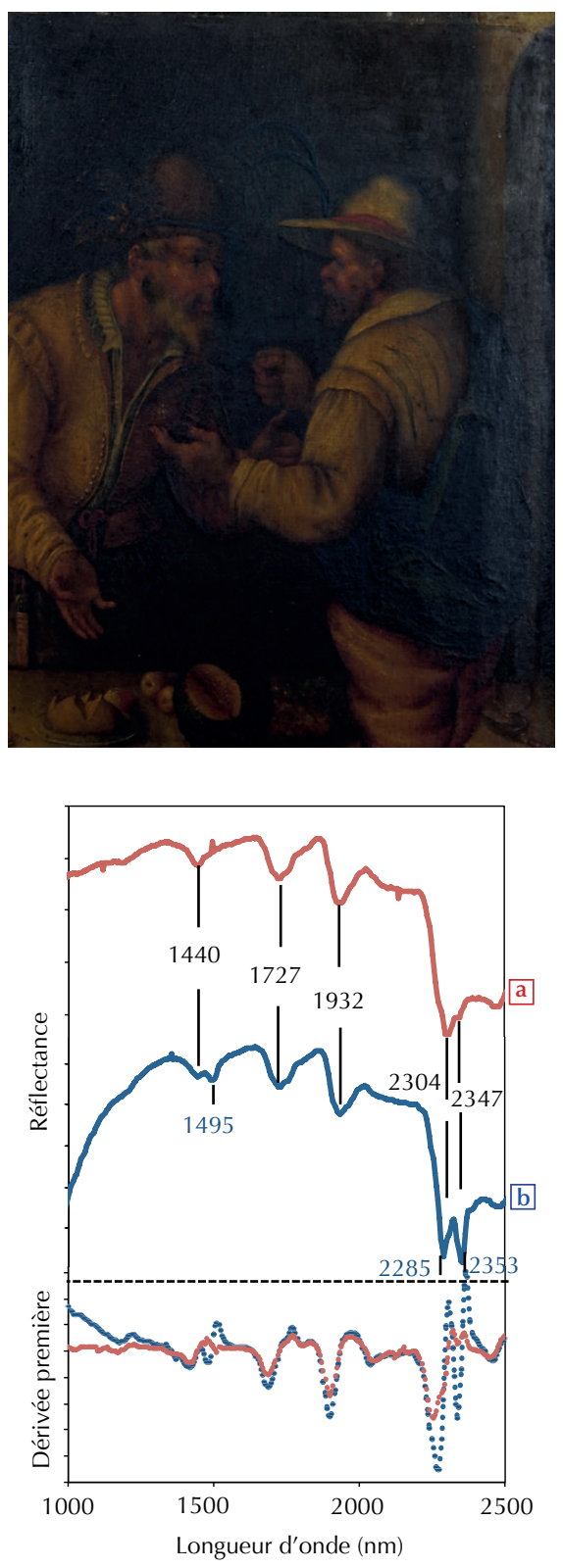
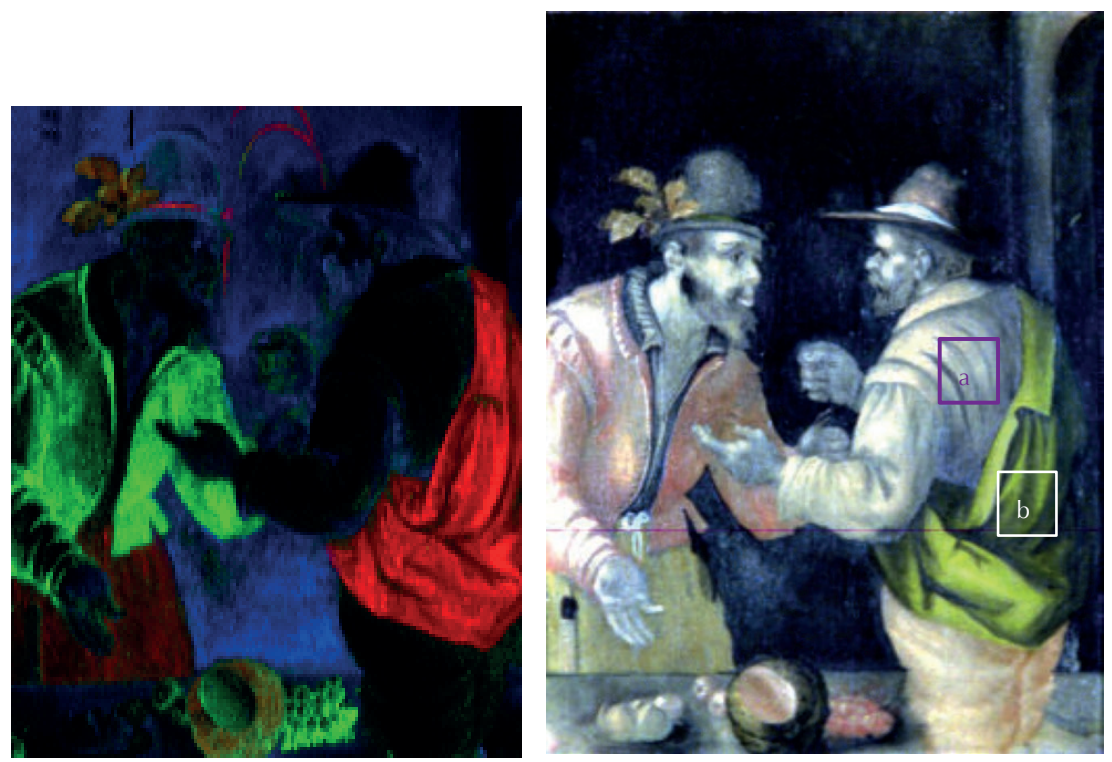

4. Imagerie d'une œuvre de l'entourage de Goltzius, « Le marchand de fruits » (collection privée, fin du XVIe siècle).

En haut, à gauche : photographie de l'œuvre. Au centre : image en fausses couleurs par fluorescence $X$ (en rouge, le cuivre Cu ; en vert, l'étain Sn et, en bleu, le fer Fe). À droite : imagerie hyperspectrale en fausses couleurs ( $\mathrm{R}: 1499 \mathrm{~nm}, \mathrm{G}: 1938 \mathrm{~nm}, \mathrm{~B}: 2353 \mathrm{~nm}$ ). Les encadrés a (violet) et b (blanc) représentent les zones d'intérêt analysées, dont les spectres de réflectance et les dérivées premières sont reportés dans la figure du bas.

On note, dans les deux zones analysées, des bandes de vibration caractéristiques $d^{\prime}$ une huile siccative avec la combinaison des bandes de vibrations de stretching $v$ et de bending $\delta$ des $\mathrm{CH}$, qui apparaissent dans la région $2300-2350 \mathrm{~nm}$, ainsi qu'à $1440 \mathrm{~nm}$. Les harmoniques des bandes de vibrations de stretching des $\mathrm{CH}_{2}$ apparaissent autour de $1727 \mathrm{~nm}$; celle de l'ester est observée à $1932 \mathrm{~nm}$. Le spectre de la zone (b) (manteau du marchand) présente en plus les bandes de vibration caractéristiques de l'azurite : bandes de vibration des groupes $\mathrm{OH}$ et $\mathrm{CO}_{3}$ à $1495 \mathrm{~nm}$ $(2 v(\mathrm{OH}))$, à $2285((v+\delta)-\mathrm{OH})$ et à $2352 \mathrm{~nm}\left(3 v\left(\mathrm{CO}_{3}\right)\right)$.

Instrumentation. Pour le proche infrarouge (1000-2500 nm), une caméra SWIR, fabriquée par la société Specim (Finlande) et équipée d'un spectrographe N25E a été utilisée ; la taille de l'image est de $384 \times 288$ pixels et la résolution spectrale est de $12 \mathrm{~nm}$. L'imagerie par fluorescence $X$ est un système conçu et réalisé au laboratoire, monté sur des platines de déplacement; I'acquisition a été obtenue avec un faisceau de $1 \mathrm{~mm}$, un pas de $1 \mathrm{~mm}$ et une durée de $500 \mathrm{~ms}$ par pixel.

\section{Conclusion}

La mise en œuvre de telles méthodes d'imagerie in situ des œuvres d'art permet d'obtenir des données sur la nature chimique des produits employés par les artistes. Rapides, faciles à mettre en œuvre, elles conduisent aujourd'hui à une meilleure compréhension de certains aspects du processus créatif, tout en aidant à préciser les phénomènes d'altération qui ont pu induire des modifications des couleurs, et en offrant des informations souvent indispensables dans le cadre des recherches sur l'originalité ou l'attribution d'une ouvre d'art.

\section{Références}

1- S. Legrand et al., Heritage Science 2 (2014) 13.

2• J. Dik et al., Anal. Chem. 80 (2008) 6436-6442.

3- Ph. Walter, L. de Viguerie et J. Castaing, Images de la physique (2011) 79-85.

4・ www.sfpt.fr/hyperspectral/?page_id=168

5• B. Valeur, La couleur dans tous ses éclats, Belin (2005).

$6 \bullet$ www.specim.fi/

7• J. K. Delaney et al., Appl. Spectrosc. 64 (2010) 584-594.

8• K. A. Dooley et al., Analyst 138 (2013) 4838. 